\title{
The Trace Amine-Associated Receptor I Agonist RO5256390 Blocks Compulsive, Binge-like Eating in Rats
}

\author{
Antonio Ferragud ${ }^{1,4}$, Adam D Howell ${ }^{1,4}$, Catherine F Moore ${ }^{1,2}$, Tina L Ta', Marius C Hoener ${ }^{3}$, \\ Valentina Sabino' and Pietro Cottone*,i \\ 'Laboratory of Addictive Disorders, Departments of Pharmacology and Experimental Therapeutics and Psychiatry, Boston University School of \\ Medicine, Boston, MA, USA; ${ }^{2}$ Graduate Program for Neuroscience, Boston University School of Medicine, Boston, MA, USA; ${ }^{3}$ Neuroscience, \\ Ophthalmology and Rare Diseases Discovery \& Translational Area, pRED, Roche Innovation Center Basel, F. Hoffmann-La Roche Ltd, Basel, \\ Switzerland
}

\begin{abstract}
Compulsive, binge eating of highly palatable food constitutes a core feature of some forms of obesity and eating disorders, as well as of the recently proposed disorder of food addiction. Trace amine-associated receptor I (TAARI) is a highly conserved G-protein-coupled receptor bound by endogenous trace amines. TAARI agonists have been shown to reduce multiple behavioral effects of drugs of abuse through their actions on the mesocorticolimbic system. In this study, we hypothesized that TAARI may have a role in compulsive, bingelike eating; we tested this hypothesis by assessing the effects of a TAARI agonist, RO5256390, in multiple excessive feeding-related behaviors induced by limiting access to a highly palatable diet in rats. Our results show that RO5256390 blocked binge-like eating in rats responding I h per day for a highly palatable sugary diet. Consistent with a palatability-selective effect, drug treatment selectively reduced the rate and regularity of palatable food responding, but it did not affect either baseline intake or food restriction-induced overeating of the standard chow diet. Furthermore, RO5256390 fully blocked compulsive-like eating when the palatable diet was offered in an aversive compartment of a light/dark conflict box, and blocked the conditioned rewarding properties of palatable food, as well as palatable foodseeking behavior in a second-order schedule of reinforcement. Drug treatment had no effect on either anxiety-like or depressive-like behavior, and it did not affect control performance in any of the tests. Importantly, rats exposed to palatable food showed decreased TAARI levels in the medial prefrontal cortex (mPFC), and RO5256390 microinfused into the infralimbic, but not prelimbic, subregion of the mPFC-reduced binge-like eating. Altogether, these results provide evidence for TAARI agonism as a novel pharmacological treatment for compulsive, binge eating.

Neuropsychopharmacology (2017) 42, |458-|470; doi:I0.1038/npp.2016.233; published online 2 November 2016
\end{abstract}

\section{INTRODUCTION}

Binge eating constitutes a core feature of forms of obesity and eating disorders (eg binge eating disorder, bulimia nervosa, and anorexia nervosa of the binge/purge type), as well as of the recently proposed disorder of food addiction (APA, 2013; Gearhardt et al, 2014). During a typical binge eating episode, individuals consume large amounts of highly processed, palatable foods in short periods of time, resulting in a very rapid eating behavior (APA, 2013; Micioni Di Bonaventura et al, 2014; Murray et al, 2014). Binge eating episodes also occur when not feeling physically hungry and can be triggered by conditioned environmental stimuli, even

*Correspondence: Dr P Cottone, Laboratory of Addictive Disorders, Departments of Pharmacology and Experimental Therapeutics and Psychiatry, Boston University School of Medicine, 72 E Concord Street, R-618, Boston, MA 02118, USA, Tel: +1 617638 5662, Fax: + I 617638 5254, E-mail: cottone@bu.edu

${ }^{4}$ These authors contributed equally to this work.

Received 14 June 2016; revised 28 September 2016; accepted 30 September 2016; accepted article preview online 6 October 2016 in absence of food per se (Giuliano and Cottone, 2015). Binge eaters experience lack of control over eating, an attribute which underlines the compulsive nature of this maladaptive feeding behavior (APA, 2013).

Trace amine-associated receptor 1 (TAAR1) is a G-protein-coupled receptor bound and activated by trace amines, a group of endogenous amines related to classical neurotransmitters, such as dopamine and serotonin (Borowsky et al, 2001; Bunzow et al, 2001; Burchett and Hicks, 2006; Grandy et al, 2016). TAAR1 is the only subtype of the TAAR family (TAAR1-9) to be phylogenetically conserved in all species studied, including humans, and it is sensitive to all trace amines (Borowsky et al, 2001; Bunzow et al, 2001; Lindemann and Hoener, 2005).

Growing evidence suggests that TAAR1 has a major role in regulating the behavioral actions of drugs of abuse, in particular those of psychostimulants. Indeed, both gene deletion and pharmacological studies have shown that TAAR1 activation reduces the reinforcing and rewarding effects of cocaine and methamphetamine, their locomotor stimulant effects, as well as reinstatement of seeking behavior 
(Harkness et al, 2015; Liu et al, 2016; Pei et al, 2015; Sukhanov et al, 2016; Thorn et al, 2014; Wolinsky et al, 2007). These actions are thought to occur via a TAAR1mediated negative modulation of dopaminergic transmission in the mesocorticolimbic system (Espinoza et al, 2015; Miller et al, 2005; Xie et al, 2007); particularly, lack of TAAR1 has been shown to result in an elevated dopaminergic firing rate, whereas TAAR1 activation suppresses such firing (Bradaia et al, 2009; Lindemann et al, 2008; Revel et al, 2011). As a result, TAAR1 agonism has been proposed as a potential therapeutic option for addictive disorders (Pei et al, 2016).

On the basis of the above described observations, as well as the well-established role of dopaminergic transmission in food reward (Michaelides et al, 2012), we hypothesized that TAAR1 agonism may reduce compulsive, binge-like eating. To test this hypothesis, we first assessed whether the systemic activation of TAAR1 would block the excessive feeding-related behaviors induced by limiting access to highly palatable food in rats (Blasio et al, 2014; Velazquez-Sanchez et al, 2014). Next, we determined the role played by the mesocorticolimbic TAAR1 system in excessive eating of palatable food first by measuring TAAR1 expression levels in selected brain regions (ie, medial prefrontal cortex (mPFC), dorsal striatum, and nucleus accumbens) and, then, based on the information acquired, by site-specifically microinfusing the TAAR1 agonist into the relevant brain regions (ie, infralimbic (IL) and prelimbic (PrL) subregions of the mPFC).

\section{MATERIALS AND METHODS}

\section{Subjects}

One hundred sixty four male Wistar rats, 45-day old, and triple-housed upon arrival (200-225 g; Charles River, Wilmington, MA), were housed in a 12:12 h reverse light cycle (lights off at 1100 hours), in a humidity- and temperaturecontrolled vivarium. Rats were given access to corn-based chow (Harlan Teklad LM-485 Diet 7012 (65\% (kcal) carbohydrate, $13 \%$ fat, $21 \%$ protein, $341 \mathrm{cal}$ per $100 \mathrm{~g}$ ); Harlan, Indianapolis, IN) and water ad libitum, unless otherwise specified. Experimental procedures were performed during rats' active cycle (dark cycle). Procedures adhered to the National Institutes of Health Guide for the Care and Use of Laboratory Animals and were approved by Boston University Medical Campus Institutional Animal Care and Use Committee (IACUC).

\section{Drugs}

The highly selective TAAR1 full agonist RO5256390 (Revel et al, 2012) was synthesized by F. Hoffman-La Roche. Doses for Experiment $1(0,1,3,10 \mathrm{mg} / \mathrm{kg})$ were based on a previously published report (Pei et al, 2014). In the other experiments, the most effective dose from Experiment 1 was used. For intracranial administration, RO5256390 was administered at the doses of $0,1.5,5$ and $15 \mu \mathrm{g}$ per side; these doses were chosen based on preliminary observations obtained in our laboratory. For intraperitoneal (i.p.) administration, RO5256390 was freshly dissolved in $0.3 \%$ Tween 80 and $0.9 \%$ saline; for intracranial administration, RO5256390 was freshly dissolved in a mixture of ethanol: cremophor:saline (2:2:18 ratio). For within-subject drug testing, 2-4 intervening days were allowed, until subjects' performance returned to baseline conditions. All drug treatments were counterbalanced, unless otherwise specified.

\section{Apparatus for Self-administration Procedures}

The individual operant test chambers (Med Associates Inc., St Albans, VT) had a grid floor and were located in ventilated, sound-attenuating enclosures (Cottone et al, 2012; Smith et al, 2015). Food reinforcers were delivered by a pellet dispenser into a head entry magazine and water reinforcers by a solenoid into a head entry liquid cup magazine. Two retractable levers were placed on the opposite wall of the chamber. $28 \mathrm{~V}$ stimulus cue-lights were located above each lever and above the food magazine. In all the procedures, pellet acquisition occurred following a nose poke response, except for the food seeking experiment in which food pellets delivery occurred following responding on a lever based on a second order schedule of reinforcement.

\section{Operant Binge-like Eating Procedure in Ad libitum-fed Rats}

Training. As previously described (Blasio et al, 2014; Velazquez-Sanchez et al, 2014), rats were allowed to selfadminister food pellets (45-mg precision pellets, 5TUM: $65.5 \%$ (kcal) carbohydrate, $10.4 \%$ fat, $24.1 \%$ protein, $330 \mathrm{cal}$ per $100 \mathrm{~g}$; TestDiet, Richmond, IN) and water (100 $\mu \mathrm{l})$ during daily $1 \mathrm{~h}$ experimental sessions under a Fixed Ratio 1 (FR1) schedule of reinforcement in the operant chambers; head entry within the food magazine was detected by a photobeam, which resulted in the delivery of a food pellet within the same food magazine. A $1.0 \mathrm{sec}$ timeout period, where no additional pellets were delivered, was used. Spillage is negligible $(4.8 \%$ of total responses, $n=23)$. At the end of the self-administration procedure, rats were returned to their home cages and fed with the same standard chow diet provided in the operant procedure, but in a $5 \mathrm{~g}$ format Cottone et al, 2012.

Escalation of palatable food intake. After stable baseline training performance was achieved, rats were divided into a Chow control group, which received the same 45-mg chow pellets offered during the training phase, and a Palatable group, which received a nutritionally complete, chocolateflavored, high sucrose $(50 \% \mathrm{kcal})$ AIN-76A-based diet (45-mg precision pellets, chocolate-flavored, 5TUL: $66.7 \%$ (kcal) carbohydrate, $12.7 \%$ fat, $20.6 \%$ protein, metabolizable energy $344 \mathrm{cal}$ per $100 \mathrm{~g}$; formulated as $45-\mathrm{mg}$ precision food pellets; TestDiet). It was previously shown that the 5TUL diet is strongly preferred over the standard chow diet by all rats (91.2\% \pm 3.7 preference; Cottone et al, 2008, 2009).

Experiment 1: effects of the TAAR1 agonist RO5256390 on operant binge-like eating. Rats in the Chow and Palatable food groups ( $n=12$ per group) were administered the TAAR1 agonist $\operatorname{RO} 5256390$ (0, 1, 3, $10 \mathrm{mg} / \mathrm{kg}$, i.p.) $30 \mathrm{~min}$ prior to the operant sessions.

\section{High Rate of Responding for Standard Chow Induced by Food Restriction}

To reach a higher rate of responding for chow during the operant sessions, rats were food restricted in their home 
cages for 12 days (to reach a total daily intake equal to $70 \%$ of a rat's daily intake; Cottone et al, 2012).

Experiment 2: effects of the TAAR1 agonist RO5256390 on high rate of responding for standard chow induced by food restriction. Food-restricted rats $(n=9$ per group) were administered the TAAR1 agonist RO5256390 (0, $10 \mathrm{mg} / \mathrm{kg}$, i.p.) $30 \mathrm{~min}$ prior to the operant sessions.

\section{Rate and Regularity of Sustained Eating: Inter-feeding Intervals and Return Map Analyses}

Inter-feeding interval analysis. To identify between group differences in the rate and regularity of sustained eating, analysis of the ln-transformed duration of consecutive interfeeding intervals (IFIs) was performed (Cottone et al, 2012). IFI, defined as the time between two consecutive food nosepoke responses, is a variable inversely correlated to eating rate, and it was automatically recorded by the computer. The mean, total IFI time, entropy, kurtosis, and skewness of the ln-transformed duration of each subject's consecutive IFIs were individually determined and then averaged across subjects.

Decreases in the IFI mean indicate a faster pellet-to-pellet responding and, therefore, an increased eating rate, which has been proposed to be an index of food palatability (Cottone et al, 2007; Iemolo et al, 2015). Decreases in the total IFI time mean a decrease in the total pellet-to-pellet time. Decreases in the histogram entropy (a measure of categorical variability, reflected in a decreased number of populated histogram bins, each with less similar event frequencies) indicate an increased regularity of intake. Conversely, an increase in the kurtosis of the IFI distribution (a measure of the distribution's 'peakedness', reflected in a more peaked top and smaller tails of the distribution) is consistent with an increased regularity of pellet-to-pellet responding. Finally, a significant increase in the skewness (a measure of the distribution's symmetry, reflected in a selective increase of the frequency of the IFI falling to the left of the histogram) is consistent with a selective increase of the fast pellet-to-pellet responding. Total IFI time was calculated as the sum of all the IFIs within a session.

Return map analysis. For return map analysis, each IFI in the time series was scatter-plotted against its subsequent IFI $(\mathrm{IFI}+1)$ in a Cartesian plane (Cottone et al, 2007; Iemolo et al, 2015).

Experiment 3: effects of the TAAR1 agonist RO5256390 on rate and regularity of sustained eating. Rats in the Chow, food-restricted Chow, and Palatable food groups $(n=7-10$ per group) were administered the TAAR1 agonist RO5256390 (0, $10 \mathrm{mg} / \mathrm{kg}$, i.p.) $30 \mathrm{~min}$ prior to the operant sessions.

\section{Compulsive-like Eating of Palatable Food: Light/Dark Box Conflict Test}

In this test, a light/dark rectangular box $(50 \times 100 \times 35 \mathrm{~cm})$ was used, in which the aversive, bright compartment $(50 \times 70 \times 35 \mathrm{~cm})$ was illuminated by a 60 lux light. The dark compartment $(50 \times 30 \times 35 \mathrm{~cm})$ had an opaque cover and $\sim 0$ lux of light. The two compartments were connected by an open doorway, which allowed the subjects to move freely between the two. A shallow, metal cup containing a preweighed amount of the same food received during selfadministration (45-mg chow or 45-mg chocolate pellets for rats in the Chow or Palatable food group, respectively) was positioned in the center of the light compartment. Apparatus-naive rats were habituated to an ante-room $2 \mathrm{~h}$ prior to testing. Under normal, control conditions, eating behavior is typically suppressed when a rat is in the aversive, bright compartment; a significant increase in food intake in spite of the adverse conditions, as compared with control conditions, was operationalized as a construct of 'compulsive-like eating' (Cottone et al, 2012; Velazquez-Sanchez et al, 2014).

Experiment 4: effects of the TAAR1 agonist RO5256390 on compulsive-like eating. Rats in the Chow and Palatable food groups ( $n=21-23$ per group) were administered the TAAR1 agonist RO5256390 (0,10 mg/kg, i.p.) $30 \mathrm{~min}$ prior to the testing session, using a between-subjects design.

\section{Conditioned Food Reward: Conditioned Place Preference Test}

The conditioned place preference (CPP) procedure was performed as previously described (Velazquez-Sanchez et al, 2015).

Experiment 5: effects of the TAAR1 agonist RO5256390 on conditioned food reward. Rats in the Chow and Palatable food groups ( $n=16-22$ per group) were administered the TAAR1 agonist RO5256390 (0,10 mg/kg, i.p.) $30 \mathrm{~min}$ prior to the post-conditioning phase, using a between-subjects design.

\section{Food-seeking Behavior: Second-order Schedule of Reinforcement}

The second-order schedule of reinforcement procedure was performed as previously described (Smith et al, 2015; Velazquez-Sanchez et al, 2015).

Experiment 6: effects of the TAAR1 agonist RO5256390 on food-seeking. Rats in the Chow and Palatable food groups ( $n=6-10$ per group) were administered the TAAR1 agonist RO5256390 (0, $10 \mathrm{mg} / \mathrm{kg}$, i.p.) $30 \mathrm{~min}$ prior to the secondorder sessions.

\section{Anxiety-like Behavior: Defensive Withdrawal Test}

For this 10-min test (Cottone et al, 2009; Parylak et al, 2012), rats were placed into a withdrawal chamber (2 1 Pyrex beaker wrapped in black tape) within an open field facing the rear. Latency to first emerge (all four paws in the open field) and withdrawal time were used as indices of anxiety-like behavior, although the number of entries into the chamber was used as an index of locomotor activity (Cottone et al, 2009; Parylak et al, 2012). 
Experiment 7: effects of the TAAR1 agonist RO5256390 on anxiety-like behavior. Rats in the Chow and Palatable food groups ( $n=10-12$ per group) were administered the TAAR1 agonist $\mathrm{RO} 5256390(0,10 \mathrm{mg} / \mathrm{kg}$, i.p.) $30 \mathrm{~min}$ prior to the testing session, using a between-subjects design.

\section{Depressive-like Behavior: Forced Swim Test}

Rats were placed into a clear acrylic cylinder $(25 \mathrm{~cm}$ diameter) filled with water $\left(24 \pm 1^{\circ} \mathrm{C} ; 42 \mathrm{~cm}\right.$ deep) for 15 min during the pre-test day and 5 min during the test day $24 \mathrm{~h}$ later. Rats were administered the drug twice: at the end of the 15-min pre-test session, and again, $30 \mathrm{~min}$ prior to the test. Immobility time is a validated measure of depressive-like behavior (Seiglie et al, 2015).

Experiment 8: effects of the TAAR1 agonist RO5256390 on depressive-like behavior. Rats in the Chow and Palatable food groups ( $n=9-12$ per group) were administered the TAAR1 agonist RO5256390 (0,10 mg/kg, i.p. $)$ at the end of the 15 -min pre-test session, and again, $30 \mathrm{~min}$ prior to the test, using a between-subjects design.

\section{Western Blot}

Brain punches from a separate cohort of rats trained in the operant binge-like eating procedure were processed for western blotting as previously described (Cottone et al, 2012). Membranes were incubated overnight at $4{ }^{\circ} \mathrm{C}$ with a primary anti-TAAR1 mouse polyclonal antibody (1:5000; provided by Roche Diagnostics) and primary anti- $\beta$-tubulin mouse monoclonal antibody, (1:30 000; sc-53140, Santa Cruz Biotechnology). The anti-TAAR1 primary antibody was validated previously (Harmeier et al, 2015). Membranes were incubated at room temperature for $1 \mathrm{~h}$ with a secondary anti-mouse IgG-HRP antibody (1:5000 or 1:10 000; sc-2004, Santa Cruz Biotechnology, for TAAR1 and $\beta$-tubulin, respectively).

Experiment 9: effects of exposure to palatable food on TAAR1 protein levels in mesocorticolimbic brain areas. $\mathrm{mPFC}$, dorsal striatum, and nucleus accumbens from rats in Chow and Palatable food groups ( $n=10-12$ per group) were compared for TAAR1 protein levels.

\section{Intracranial Surgeries, Microinfusion Procedure, and Cannula Placement}

Rats were stereotaxically implanted with bilateral, intracranial cannulas targeting the IL and the PrL subregions of the $\mathrm{mPFC}$, as described previously (Dore et al, 2013; Sabino et al, 2007).

Experiments 10-11: effects of microinfusion of the TAAR1 agonist RO5256390 into the IL cortex and PrL cortex on operant binge-like eating. Rats in the Chow and Palatable food groups were microinfused with the TAAR1 agonist RO5256390 (0, 1.5, 5, $15 \mu \mathrm{g}$ per side) into either the IL ( $n=8-10$ per group) or the PrL ( $n=11-12$ per group) cortex $10 \mathrm{~min}$ prior to the operant sessions.

\section{Statistical Analyses}

Data were analyzed by simple or factorial ANOVAs followed by Bonferroni post hoc test. Statistical significance level was set at $p \leqslant 0.05$. The software/graphic packages used were SigmaPlot 11.0, Statistica 7.0, and Origin 8.5.

\section{RESULTS}

\section{Experiment 1: Effects of the TAAR1 Agonist R05256390 on Operant Binge-like Eating}

Rats, initially trained to respond for the standard chow diet, were split in two matched groups and assigned to either the Chow or the Palatable condition $(M \pm$ SEM of last four selfadministration sessions, $18.6 \pm 1.3$ and $18.8 \pm 0.8 \mathrm{kcal}$ for Chow and Palatable respectively, $\mathrm{t}_{22}=0.13, p=0.897$, not shown). Rats allowed to self-administer the sugary, highly palatable diet $1 \mathrm{~h}$ per day markedly escalated food intake, unlike the control Chow group, whose intake remained stable across the 15 days of observation (Diet, $F_{1,22}=49.83$, $p=0.001$; Diet $\times$ Day, $\mathrm{F}_{14,31}=16.95, p=0.001$; Figure 1a).

When the effects of the TAAR1 agonist RO5256390 on food intake were tested, results showed that rats from the Palatable food group consumed significantly more food compared with the Chow controls under vehicle conditions (Diet, $\mathrm{F}_{1,22}=62.72, p=0.001$; Figure $1 \mathrm{~b}$ ). Drug treatment selectively and dose dependently blocked the binge-like eating of the Palatable food group in the operant task, without affecting intake of the control Chow group (Dose, $\mathrm{F}_{3,66}=22.17, p=0.001$; Diet $\times$ Dose, $\mathrm{F}_{3,66}=10.02, p=0.001$ ). In rats fed with the Palatable diet, RO5256390 treatment significantly decreased palatable food responding, compared with vehicle, at all doses tested. At the highest dose tested $(10 \mathrm{mg} / \mathrm{kg})$, drug treatment fully blocked binge-like eating, as treated rats' intake in the Palatable food group did not differ from either Chow/Vehicle or Chow/RO5256390 $10 \mathrm{mg} / \mathrm{kg}$. Figure $1 \mathrm{c}$ shows the time course of responding (Diet $\times$ Dose, $\left.\mathrm{F}_{3,66}=3.41, p=0.022\right)$.

Experiment 2: Effects of the TAAR1 Agonist R05256390 on High Rate of Responding for Standard Chow Induced by Food Restriction

Figure 1d shows the escalation of the standard chow responding during daily home-cage food restriction (Day, $\mathrm{F}_{11,88}=30.17, p=0.001$ ). Responding for the standard chow diet in food-restricted rats was comparable to the responding of vehicle-treated ad libitum-fed rats in the Palatable food group in the RO5256390 administration FR1 study $\left(t_{20}=0.21\right.$, $p=0.832$ ). Systemic treatment with the $10 \mathrm{mg} / \mathrm{kg}$ dose of RO5256390 had no effect on the high rate of responding for standard chow in the operant FR1 food intake task in foodrestricted rats $\left(t_{8}=0.53, p=0.610\right.$; Figure $\left.1 c\right)$.

\section{Experiment 3: Effects of the TAAR1 Agonist R05256390 on Rate and Regularity of Sustained Eating}

As shown in Figure 2, vehicle-treated binge-eating rats in the Palatable food group showed a significantly smaller IFI mean, but no different total IFI time compared with ad libitum-fed rats in the Chow food group; surprisingly, 


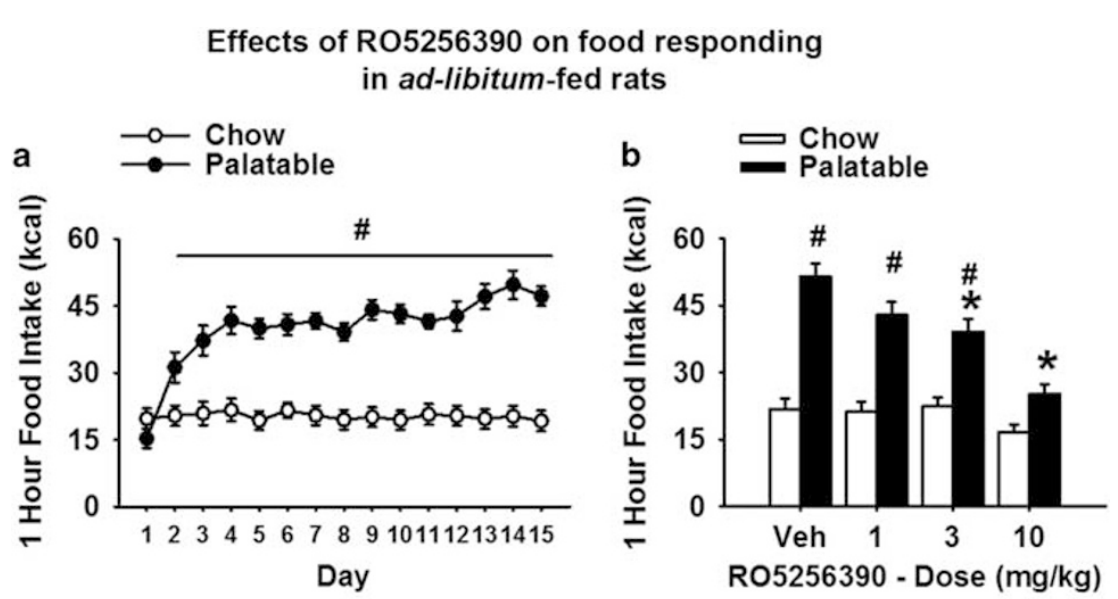

C

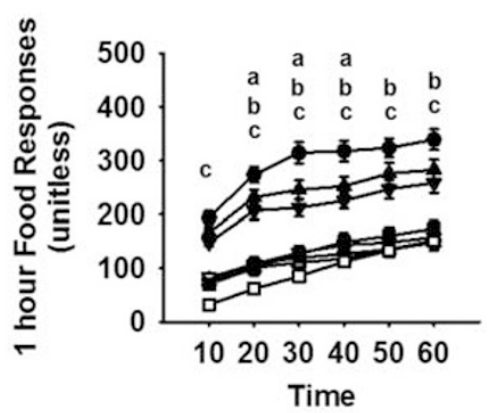

-0- Chow / Vehicle

$\neg$ Chow/ RO5256390 $1 \mathrm{mg} / \mathrm{kg}$

$\rightarrow-$ Chow / RO5256390 $3 \mathrm{mg} / \mathrm{kg}$

$\longrightarrow$ - Chow / RO5256390 10 mg/kg

$\rightarrow$ Palatable / Vehicle

—— Palatable / RO5256390 $1 \mathrm{mg} / \mathrm{kg}$

$\rightarrow$ Palatable / RO5256390 $3 \mathrm{mg} / \mathrm{kg}$

$\rightarrow$ Palatable / RO5256390 $10 \mathrm{mg} / \mathrm{kg}$

Effects of RO5256390 on food responding in food restricted rats
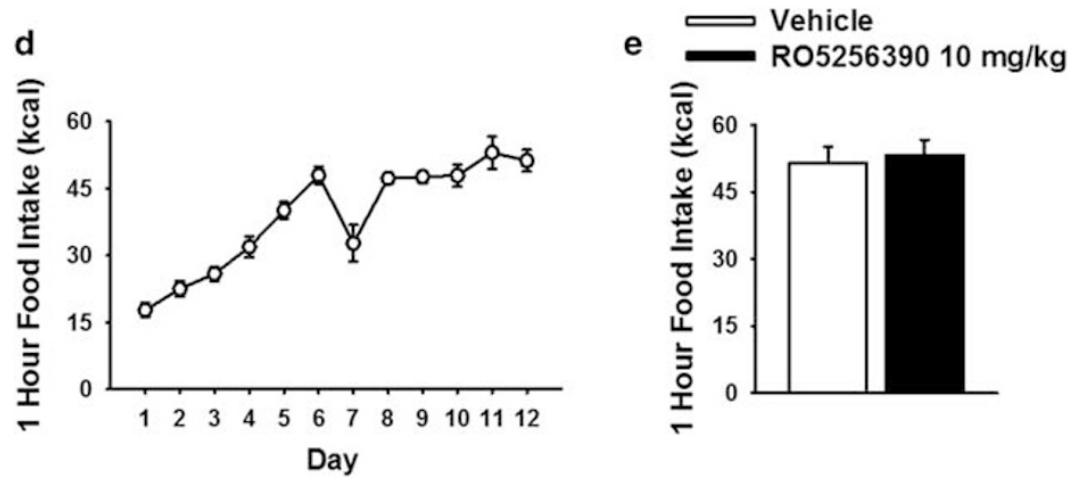

Figure I TAARI activation blocks binge-like eating without affecting either chow intake or food restriction-induced chow overeating. (a) Effects of daily I $\mathrm{h}$ self-administration of either a standard chow or a highly palatable diet on food responding in male Wistar rats $(n=12$ per group). (b) Effects of systemic administration of the TAARI agonist RO5256390 (0, I, 3, $10 \mathrm{mg} / \mathrm{kg}$, i.p.) on I h food self-administration of either a standard chow or a highly palatable diet ( $n=12$ per group). (c) Time course of food responding. (d) Effects of food restriction on daily I h self-administration of a standard chow diet on food responding in male Wistar rats ( $n=9$ per group). (e) Effects of systemic administration of the TAARI agonist RO5256390 (0, $10 \mathrm{mg} / \mathrm{kg}$, i.p.) on high rate of responding for standard chow induced by food restriction. Panels represent $M \pm S E M$. ${ }^{*} p \leqslant 0.05$ vs Chow (a) or Vehicle Chow (b); * $p \leqslant 0.05$ vs Vehicle Palatable; (a) RO5256390 I mg/kg Palatable vs Vehicle Palatable $p \leqslant 0.05$; (b) RO5256390 3 mg/kg Palatable vs Vehicle Palatable $p \leqslant 0.05 ;$ (c) RO5256390 $10 \mathrm{mg} / \mathrm{kg}$ Palatable vs Vehicle Palatable $p \leqslant 0.05$

vehicle-treated food-restricted chow rats showed a similar IFI mean but a much higher total IFI time than control ad libitum-fed rats in the Chow food group (IFI mean: Diet, $\mathrm{F}_{2,23}=18.78, p=0.001$; total IFI time: Diet, $\mathrm{F}_{2,23}=20.12$, $p=0.001$; Figure $2 \mathrm{a}$ and $\mathrm{b}$ ). Therefore, even though both Palatable and food-restricted group rats ate significantly more pellets than control ad libitum-fed rats in the Chow food group (Diet, $\mathrm{F}_{2,23}=37.71, p=0.001$; not shown), rats fed with the palatable diet consumed food at a higher rate within a similar time, while food-restricted chow rats ate at a similar rate but in a longer time. In addition, both rats in the Palatable food group and food-restricted chow rats showed an increased regularity of sustained eating as revealed by a decreased entropy, compared with ad libitum-fed Chow controls (entropy: Diet, $\mathrm{F}_{2,23}=49.85, p=0.001$; Figure $2 \mathrm{c}$ ). However, although rats' IFI distribution in the Palatable food group was more skewed and leptokurtic than that of ad libitum-fed rats in the Chow food group, the shape of the 
Effects of RO5256390 on inter-feeding intervals (IFIs) and return map

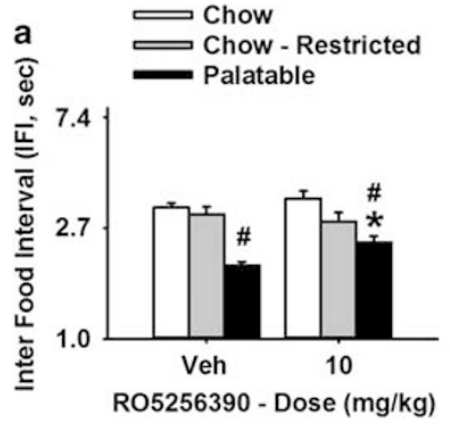

b

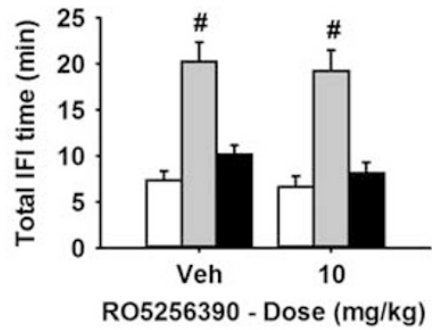

C

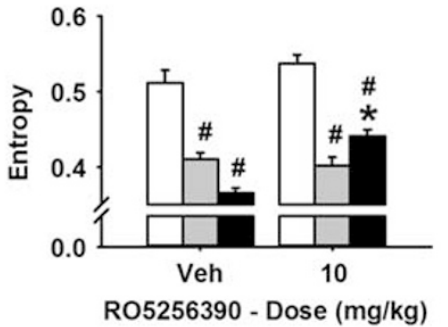

Chow - Restricted Vehicle

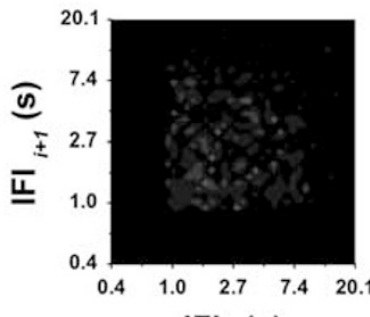

$\mathrm{IFI}_{i}(\mathrm{~s})$
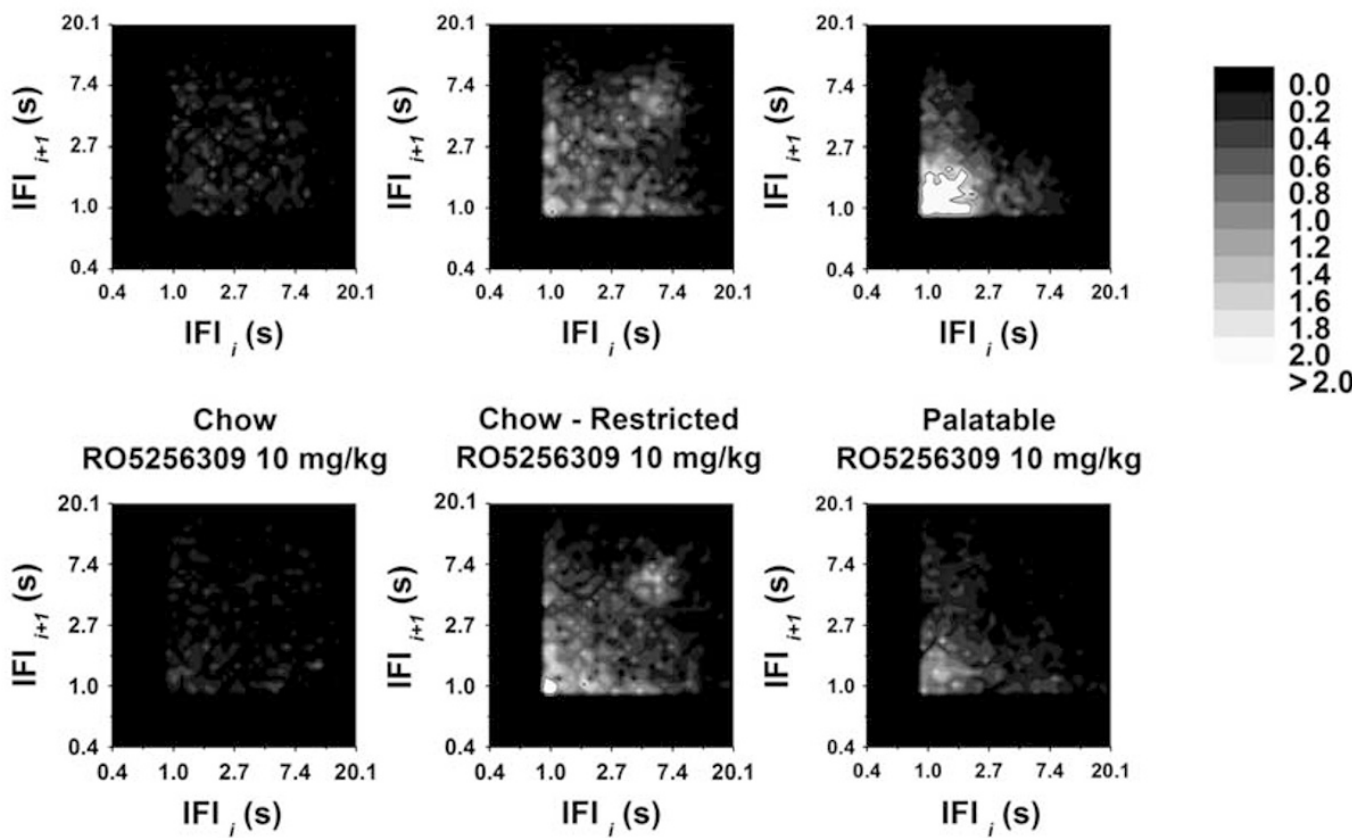

Palatable RO5256309 $10 \mathrm{mg} / \mathrm{kg}$
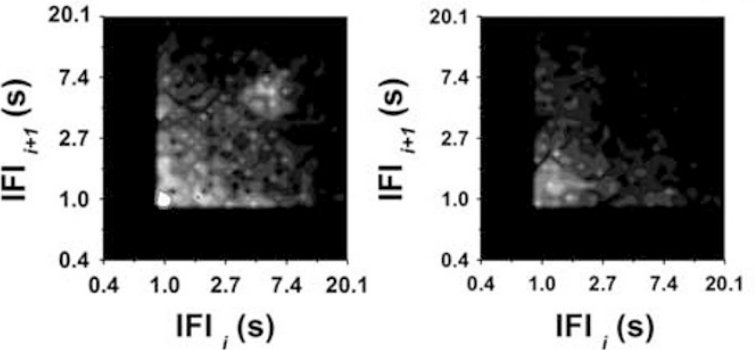

Figure 2 TAARI activation reduces the rate and the regularity of responding in binge eating rats. Effects of systemic administration of the TAARI agonist RO5256390 (0, 10 mg/ $/ \mathrm{kg}$, i.p.) on (a) inter-feeding interval (IFI) mean, (b) total IFI time, and (c) entropy, in daily I h self-administration of either a standard chow (in ad libitum or food restricted regimens) or a highly palatable diet in male Wistar rats ( $n=7-10$ per group). (d) Return map of normalized probability density plots representing successive IFIs from all subjects. Increasing gray-scale intensity (from white to black; or color intensity from black to red in the color figure) represents increasing probabilities (from 0 to 2) of successive eating events and, therefore, increased regularity of eating from pellet-to-pellet occurring at the corresponding IFI duration. Panels represent $M \pm$ SEM. ${ }^{\#} p \leqslant 0.05$ vs Vehicle Chow; * $p \leqslant 0.05$ vs Vehicle Palatable. A full color version of this figure is available at the Neuropsychopharmacology journal online.

distribution of IFIs in food-restricted chow rats was no different than controls (skewness: Diet, $F_{2,23}=4.55$, $p=0.022$; kurtosis: Diet, $\mathrm{F}_{2,23}=3.51, p=0.047$; Supplementary Figure S1A and B). In the return map analysis, when compared with the homogeneous distribution across a wide range of IFI values of Chow controls, food responding of vehicle-treated rats in the Palatable food group was characterized by a denser cluster of IFIs skewed in the bottom left of the map, while responding in food-restricted chow rats was characterized by an overall increase of events across the entire range of IFIs, with sporadic clustered events (Figure 2d).

When RO5256390 (10 mg/kg) was administered, only Palatable food group responding was affected by drug treatment. Indeed, although the drug treatment had no effect on either ad libitum-fed Chow rats or food-restricted chow rats, rats in the Palatable food group RO5256390 increased the IFI mean (Dose, $\mathrm{F}_{1,23}=5.82, p=0.024$; Diet $\times$ Dose, $F_{2,23}=7.34, p=0.003$; Figure $2 \mathrm{a}$ ) and increased entropy (Dose, $\quad \mathrm{F}_{1,23}=28.91, \quad p=0.001 ; \quad$ Diet $\times$ Dose, $\mathrm{F}_{2,23}=24.16, p=0.001$; Figure 2c). Post hoc comparisons revealed that drug treatment did not meaningfully affect total IFI time, skewness, or kurtosis (total IFI time: Dose, $\mathrm{F}_{1,23}=5.24, \quad p=0.032 ;$ Diet $\times$ Dose, $\mathrm{F}_{2,23}=0.59, p=0.562$; skewness: Dose, $\mathrm{F}_{1,23}=1.58, \quad p=0.221$; Diet $\times$ Dose, $\mathrm{F}_{2,23}=4.99, p=0.016$; kurtosis: Dose, $\mathrm{F}_{1,23}=0.55, p=0.467$; Diet $\times$ Dose $, \quad F_{2,23}=1.39, \quad p=0.268$; Figure $2 b$ and Supplementary Figure S1A and B). As already observed in Experiment 1, RO5256390 decreased the number of pellets eaten only in the Palatable food group (Dose, $\mathrm{F}_{1,23}=12.84$, $p=0.002$; Diet $\times$ Dose, $\mathrm{F}_{2,23}=10.74, p=0.001$, not shown). In the return map analysis (Figure $2 \mathrm{~d}$ ), the effects of 


\section{Effects of RO5256390 on Light/Dark Box Conflict Test}

a

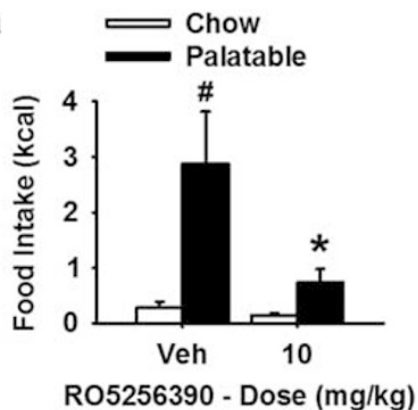

Effects of RO5256390 on Conditioned Place Preference

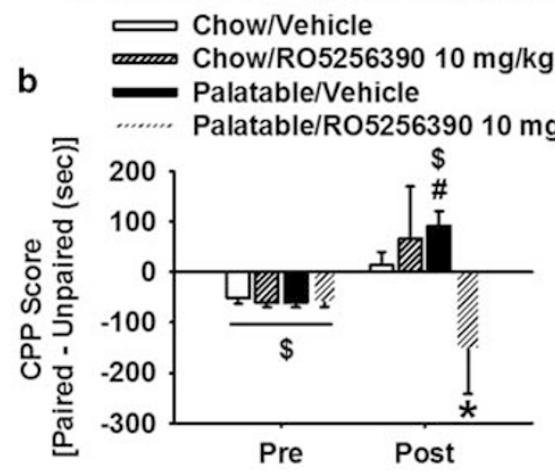

Effects of RO5256390 on Second-Order Schedule of Reinforcement

C
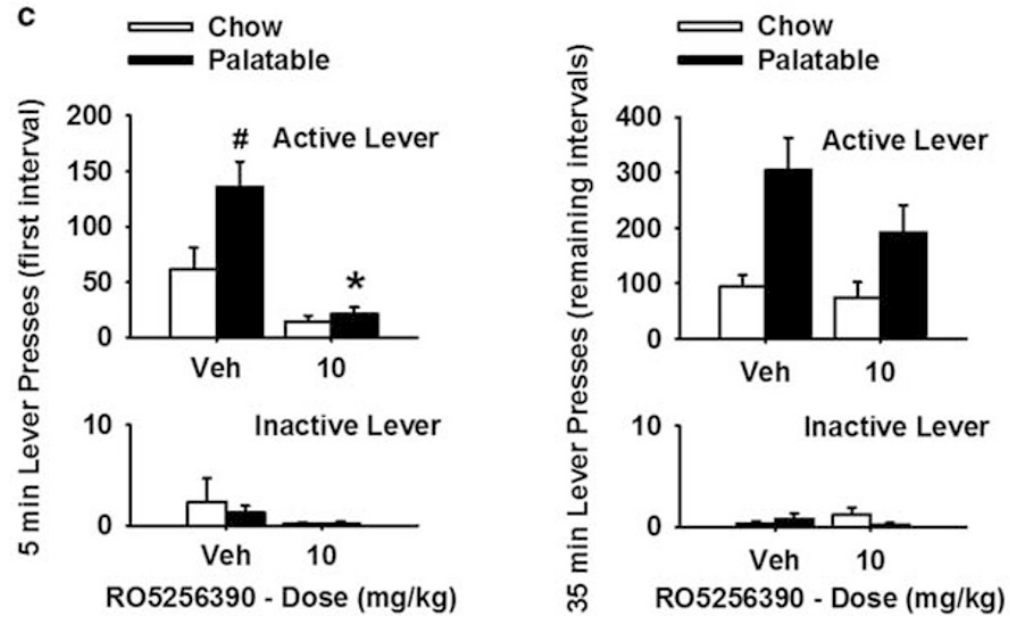

Figure 3 TAARI activation blocks palatable food-driven compulsive eating, conditioned food reward, and food-seeking of palatable food in binge eating rats. Effects of systemic administration of the TAARI agonist RO5256390 (0, $10 \mathrm{mg} / \mathrm{kg}$, i.p.) on (a) food intake during the light/dark conflict test ( $n=21-23$ per group), (b) CPP score ( $n=16-22$ per group), (c) active and inactive lever presses during the first interval (left) and the remaining intervals (right) of a secondorder schedule of reinforcement in male Wistar rats with a history of daily I h self-administration of either a standard chow or a highly palatable diet. Panels represent $M \pm$ SEM. ${ }^{*} p \leqslant 0.05$ vs Vehicle Chow; ${ }^{*} p \leqslant 0.05$ vs Vehicle Palatable; ${ }^{\$} p \leqslant 0.05$ vs 0.

RO5256390 treatment resulted in a more diffuse and less skewed pellet-to-pellet responding, as compared with the vehicle condition. Therefore, RO5256390 treatment tended to normalize the rate and the regularity of responding in bingeing of rats in the Palatable food group.

Experiment 4: Effects of the TAAR1 Agonist R05256390 on Compulsive-like Eating

When food was placed in the bright, aversive compartment of the light/dark apparatus, control Chow intake was suppressed. Under vehicle conditions, rats in the Palatable food group consumed 10-fold more food as compared with the control Chow group (Diet, $F_{1,83}=5.34, p=0.023$; Figure 3a (see Supplementary Figure S2 for the grams of food consumed). In addition, RO5256390, administered at the $10 \mathrm{mg} / \mathrm{kg}$ dose, fully blocked compulsive-like eating of the palatable diet (Dose, $\mathrm{F}_{1,83}=10.37, p=0.002$; Diet $\times$ Dose, $\left.\mathrm{F}_{1,83}=4.10, p=0.046\right)$, as drug-treated rats' intake in the
Palatable food group did not differ from that of Chow controls.

\section{Experiment 5: Effects of the TAAR1 Agonist R05256390} on Conditioned Food Reward

Rats in all conditions were matched to spend a similar amount of time in the least preferred compartment, as revealed by similar preconditioning CPP scores (Diet, $\mathrm{F}_{1,71}=0.27, p=0.635$; Dose, $\mathrm{F}_{1,71}=0.38, p=0.541$; Diet $\times$ Dose: $\mathrm{F}_{1,71}=0.31, p=0.577$; Figure $3 \mathrm{~b}$ ). The analysis of the post-conditioning CPP scores indicated that, under vehicle condition, rats in the Palatable, but not Chow food group, displayed place conditioning, as they spent significantly more time in the initial non-preferred compartment as compared with their score in the pre-conditioning phase (biased protocol). However, RO5256390, injected at the $10 \mathrm{mg} / \mathrm{kg}$ dose, fully blocked the expression of the place preference in rats in the Palatable food group, as their post-conditioning score did not significantly differ from their pre-conditioning 
score, although it significantly differed from the postconditioning score of vehicle-treated rats in the same group (Diet $\times$ Dose: $F_{1,71}=4.73, p=0.032$; Figure $\left.3 b\right)$.

\section{Experiment 6: Effects of the TAAR1 Agonist R05256390 on Food-Seeking}

In the second order schedule of reinforcement, the analysis of the first interval, which occurs before food ingestion, revealed that the rats in the Palatable food group showed a higher number of active lever responses compared with the Chow control rats (Diet, $\mathrm{F}_{1,14}=6.52, p=0.023$; Figure $3 c / l e f t)$. RO5256390 administration selectively blocked active lever responding in rats fed with the palatable food, but not in controls (Dose, $\mathrm{F}_{1,14}=19.74, \mathrm{p}<0.001$; Diet $\times$ Dose, $\mathrm{F}_{1,14}=3.42, p=0.086$; Figure $3 \mathrm{c} / \mathrm{left}$ ). Conversely, in the remaining intervals, which occur after food ingestion, although a main effect of Diet was observed (Diet, $\mathrm{F}_{1,14}=9.06, p=0.009$ Figure 3c/right), no effect of either Dose or interaction Diet $\times$ Dose was detected (Dose, $\mathrm{F}_{1,14}=1.85, p=0.194 ;$ Diet $\times$ Dose, $\mathrm{F}_{1,14}=0.87, p=0.365$, Figure $3 c /$ right).

Inactive lever responding was not affected by either the food provided or the drug treatment at any point during the second order session (first interval: Diet, $\mathrm{F}_{1,14}=0.26, p=0.618 ;$ Dose, $\mathrm{F}_{1,14}=2.62, p=0.128$; Diet $\times$ Dose, $F_{1,14}=0.28, p=0.605$; remaining intervals: Diet, $\mathrm{F}_{1,14}=0.20, p=0.661 ;$ Dose, $\mathrm{F}_{1,14}=0.11, p=0.741 ;$ Diet $\times$ Dose, $\mathrm{F}_{1,14}=4.27, p=0.057$; Figure $3 \mathrm{c}$ ).

\section{Experiment 7: Effects of the TAAR1 Agonist R05256390 on Anxiety-like Behavior}

When anxiety-like behavior was assessed in Chow and Palatable food groups using the defensive withdrawal test, under vehicle conditions the two diet groups did not differ in the latency to exit the sheltered chamber (Diet, $\mathrm{F}_{1,39}=0.28$, $p=0.600$; Figure $4 \mathrm{a} / \mathrm{left}$ ). In addition, latency was not affected by RO5256390 treatment (Dose, $F_{1,39}=1.07$, $p=0.600$; Diet $\times$ Dose, $\left.F_{1,39}=0.01, p=0.916\right)$. Furthermore, vehicle-treated Chow and Palatable food groups rats did not differ in the time spent in the sheltered chamber (Diet, $\mathrm{F}_{1,39}=1.64, p=0.207$; Figure $4 \mathrm{a} /$ right). Drug treatment did not affect the time spent in the sheltered chamber either (Dose, $\quad F_{1,39}=0.01, \quad p=0.981 ;$ Diet $\times$ Dose, $F_{1,39}=0.03$, $p=0.863)$. Entries to the chamber did not differ between either diet or dose groups (Diet, $\mathrm{F}_{1,39}=0.86, p=0.359$; Dose, $\mathrm{F}_{1,39}=0.06, p=0.812$; Diet $\times$ Dose, $\mathrm{F}_{1,39}=0.10, p=0.747$; not shown).

\section{Experiment 8: Effects of the TAAR1 Agonist R05256390 on Depressive-like Behavior}

When depressive-like behavior was assessed in Chow and Palatable food groups using the forced swim test, under vehicle conditions the two diet groups did not differ in immobility time (Diet, $\mathrm{F}_{1,38}=0.92, p=0.342$; Figure $4 \mathrm{~b} / \mathrm{left}$ ), swimming time (Diet, $\mathrm{F}_{1,38}=0.19, p=0.669$; Figure $4 \mathrm{~b} /$ right) or climbing time (Diet, $\mathrm{F}_{1,38}=2.96, p=0.094$; not shown). In addition, RO5256390 treatment did not affect immobility time (Dose, $F_{1,38}=0.06, p=0.806$; Diet $\times$ Dose, $F_{1,38}=0.50$, $p=0.484$; Figure $4 \mathrm{~b} / \mathrm{left}$ ), swimming time (Dose, $\mathrm{F}_{1,38}=0.04$, $p=0.848 ;$ Diet $\times$ Dose, $F_{1,38}=1.03, p=0.315 ;$ Figure $4 \mathrm{~b} /$ right), or climbing time (Dose, $\mathrm{F}_{1,38}=0.07, p=0.792$; Diet $\times$ Dose, $F_{1,38}=0.06, p=0.811$; not shown).

Experiment 9: Effects of Exposure to Palatable Food on TAAR1 Protein Levels in Mesocorticolimbic Brain Areas

TAAR1 protein levels were decreased in the $\mathrm{mPFC}$ $\left(t_{19}=2.56, p=0.019\right.$; Figure $\left.5 \mathrm{a}-\mathrm{c}\right)$, although no differences were observed in either the dorsal striatum $\left(t_{21}=0.38\right.$, $p=0.709)$ or the nucleus accumbens $\left(t_{20}=0.59, p=0.563\right)$ (Supplementary Figure S3).

\section{Experiments 10-11: Effects of Microinfusion of the TAAR1 Agonist R05256390 into the IL Cortex and PrL Cortex on Operant Binge-like Eating}

Microinfusion of the TAAR1 agonist RO5256390 into the IL subregion of the mPFC selectively and dose dependently reduced food responding in rats in the Palatable food group (Diet, $\mathrm{F}_{1,16}=94.27, p=0.001 ;$ Dose, $\mathrm{F}_{1,48}=7.09, p=0.001$; Diet $\times$ Dose, $\left.F_{1,48}=7.77, p=0.001\right)$. A significant effect was observed at both the 5 and $15 \mu \mathrm{g}$ per side doses as compared with vehicle condition $(23.0 \%$ reduction observed with the $15 \mu \mathrm{g}$ per side dose). No effect was observed in control Chow rats, Figure 5d-f). Conversely, when RO5256390 was microinfused in the PrL cortex, drug treatment exerted no effect in either Chow or Palatable food responding (Diet, $\mathrm{F}_{1,21}=32.41, p=0.001 ;$ Dose, $\mathrm{F}_{1,63}=0.58, p=0.632$; Diet $\mathrm{x}$ Dose, $F_{1,63}=1.39, p=0.253$; Supplementary Figure S4).

\section{DISCUSSION}

\section{R05256390 Blocks Binge-like Eating and Reduces the Rate of Palatable Food Intake}

In this study, we found that the selective TAAR1 agonist RO5256390 dose dependently blocked binge eating of highly palatable food induced by a $1 \mathrm{~h}$ per day limited access procedure. Drug treatment was effective at all doses tested, and the highest dose $(10 \mathrm{mg} / \mathrm{kg})$ reduced palatable food intake by $51.2 \pm 4.4 \%(M \pm S E M)$. This effect was selective for the palatable diet, as the standard chow control intake was unaffected by drug treatment. Therefore, drug effect was not secondary to an overall behavioral deficit and was specific for palatability-rather than energy homeostaticdependent food consumption.

Consistent with a palatability-selective effect, the highest dose $(10 \mathrm{mg} / \mathrm{kg})$ of RO5256390 did not affect standard chow diet overeating induced by a food restriction procedure, excluding the alternative interpretation that drug effects were dependent on a high rate of responding. Notably, our food restriction protocol was performed over 12 days before any pharmacological treatment, a time sufficient to induce neurochemical and behavioral adaptations in the reward system, which increase susceptibility to drugs of abuse (D'Cunha et al, 2013). Further evidence of a palatabilityselective effect of RO5256390 was provided by the analysis of the IFIs. As a result of the increased palatability of the diet, responding in binge eating rats was characterized by highly clustered and short IFIs, as opposed to the much more diffuse and long IFIs of chow control rats. The increased 
Effects of RO5256390 on Defensive Withdrawal
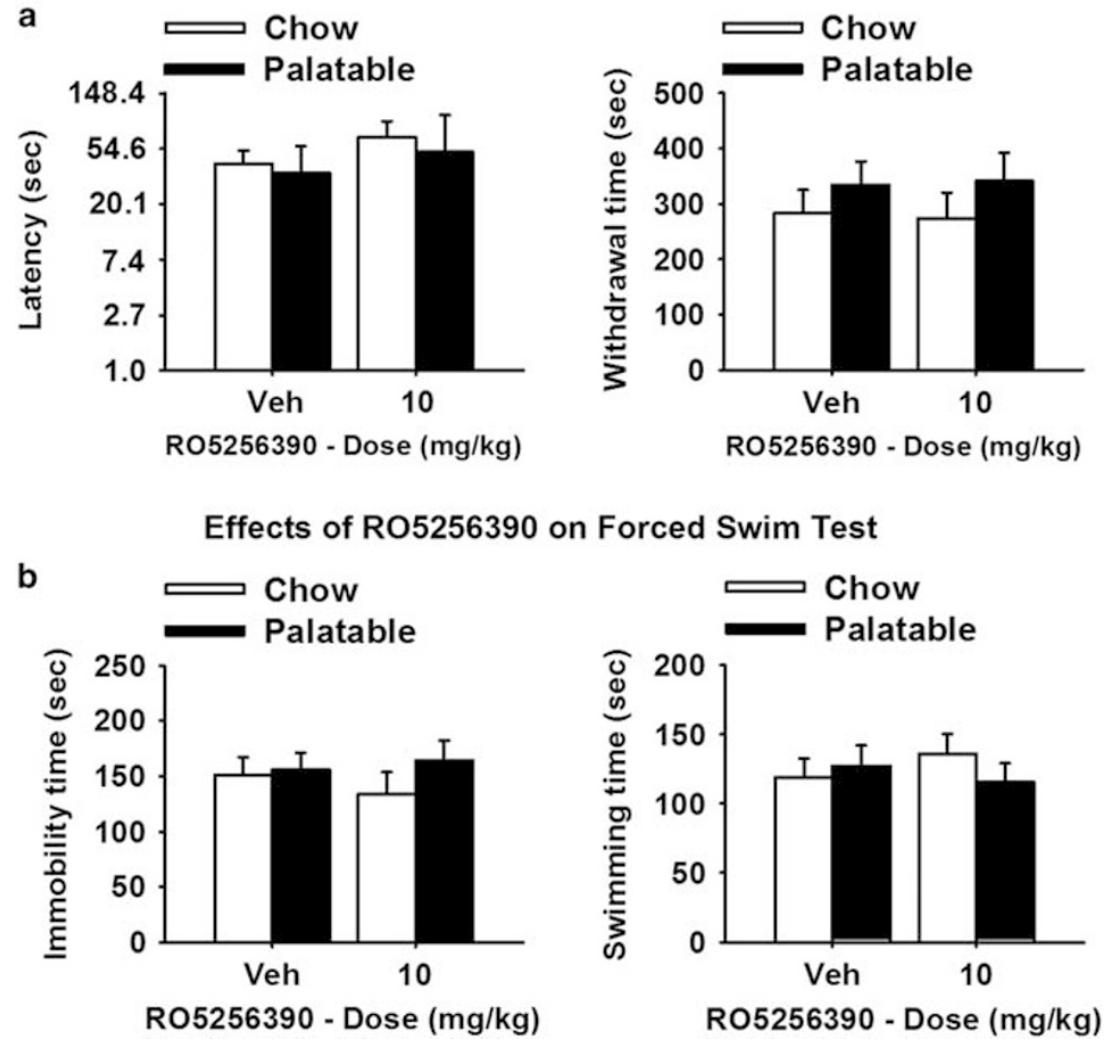

Figure 4 TAARI activation does not affect anxiety-like or depressive-like behavior in binge eating rats. Effects of systemic administration of the TAARI agonist RO5256390 (0, $10 \mathrm{mg} / \mathrm{kg}$, i.p.) on (a, left) latency to first emerge, (a, right) withdrawal time in the chamber in the defensive withdrawal test ( $n=10-12$ per group); (b, left) immobility time, (b, right) swimming time in a forced swim test ( $n=9-12$ per group), in male Wistar rats with a history of daily I h selfadministration of either a standard chow or a highly palatable diet. Panels represent $M \pm S E M$.

sustained eating rate was independent from the high rate of response, as food-restricted rats, which ate the same number of pellets as rats bingeing on the palatable diet, ate much more slowly and in a longer time frame. Treatment with RO5256390 selectively decreased the rate and the regularity of pellet-to-pellet responding in binge eating rats, without affecting responding for standard chow in either ad libitum fed or food-restricted rats. Thus, agonism of TAAR1 specifically disrupted the ability of palatable food, but not standard chow (consumed at any rate of response or in any feeding state) to sustain clustered feeding responses. Overall, these findings strengthen the concept that, despite being quantitatively equivalent, binge eating induced by an increased palatability of the food is different at a behavioral, pharmacological, and neurobiological level than overeating induced by increased energy needs due to food restriction/ deprivation (Cottone et al, 2012).

The observed decrease in palatable food responding following RO5256390 treatment is in line with previous reports obtained with full agonists of TAAR1. RO5256390 was able to decrease, as well as slow down, responding for chocolate flavored pellets at the 10 , but not the $3 \mathrm{mg} / \mathrm{kg}$ dose (Pei et al, 2014). Here we observed that the decrease in palatable food responding occurs at all the doses tested (1, 3 and $10 \mathrm{mg} / \mathrm{kg}$ ). However, in the previous study the rats' intake was capped to 40 food pellets per session (Pei et al,
2014), whereas here rats obtained an average of $332.8 \pm 19.0$ $(M \pm$ SEM $)$ palatable pellets. Furthermore, our data are consistent with a recent study showing that the TAAR1 full agonist RO5166017 was able to reduce the intake of a highfat diet in diet-induced obesity mice (Raab et al, 2016).

\section{R05256390 Blocks Compulsive-like Eating}

We observed that RO5256390 blocked compulsive-like eating behavior in a light/dark box conflict test. Compulsivity is a behavioral construct observed in multiple disorders, including addictions, as well as certain disorders of pathological eating, where maladaptive behavior perseverates in spite of medical, psychological, emotional, and social impairment (APA, 2013; Gearhardt et al, 2014). In our conditions, feeding is suppressed when the control rats face the aversive bright compartment of the light/dark box, whereas bingeing rats compulsively consume the palatable diet in face of the potentially risky circumstances (Cottone et al, 2012; Dore et al, 2014; Velazquez-Sanchez et al, 2014). TAAR1 activation fully blocked compulsive eating of palatable food, reducing intake in the bright compartment by $75.8 \pm 8.2 \%(M \pm$ SEM $)$ as compared with vehicle-treated rats in the same group. Importantly, the alternative interpretation that this effect was induced by a potential anxiogenic profile of drug treatment can be confidently excluded as RO5256390 exerted no effect on either anxiety- or depressive-like behavior. 
TAAR1 Western blot in mPFC

b

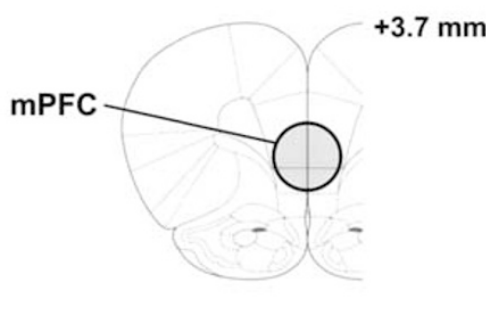

C

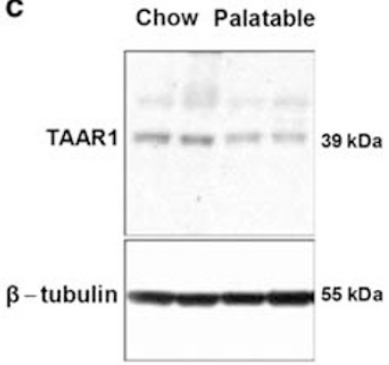

Effects of IL microinfusion of RO5256390 on food responding in ad-libitum-fed rats

d

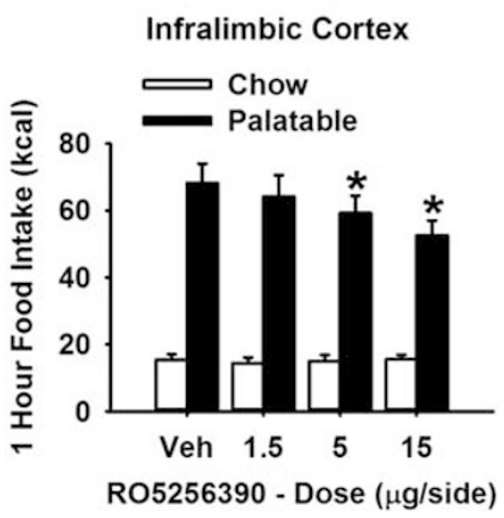

e

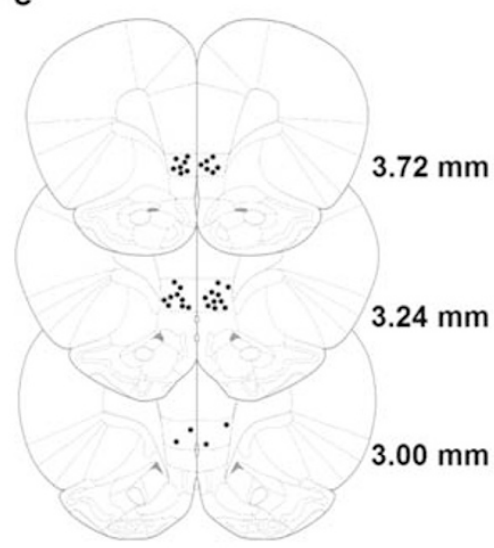

f

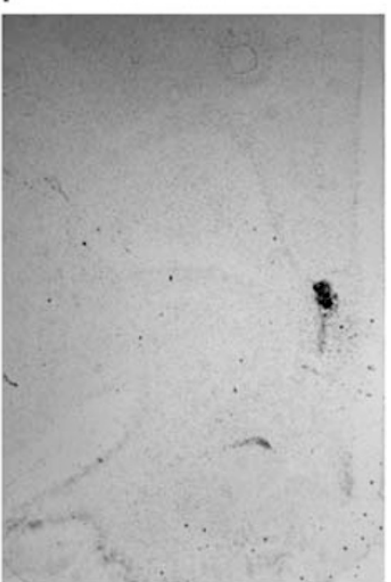

Figure 5 Limited access to palatable food decreases TAARI protein levels in the prefrontal cortex; infralimbic cortex (IL) activation of TAARI reduces binge-like eating. (a) TAARI protein levels in the mPFC of male Wistar rats self-administering I h per day either a standard chow or a highly palatable diet ( $n=10-12$ per group). (b) Drawing of rat brain slices used for western blotting studies; circle shows punch of mPFC. (c) Representative western blot of TAARI and $\beta$-tubulin. (d) Effects of microinfusion of the TAARI agonist RO5256390 (0, I.5, 5, I $5 \mathrm{\mu g}$ per side) into the infralimbic cortex (IL) subregion of the mPFC on I h food self-administration of either a standard chow or a highly palatable diet ( $n=8-10$ per group). (e) Drawing of coronal rats' brain slices; dots represent the injection sites in the $\mathrm{IL}$ included in the data analysis. Panels represent $M \pm S E M$. (f) Photomicrograph shows a coronal section of the brain of a rat with representative injection sites in the IL. Panels represent $M \pm S E M$. * $p \leqslant 0.05$ vs Chow.

\section{R05256390 Blocks the Strength of Palatable-food- associated Stimuli}

In this study, RO5256390 blocked the strength of palatablefood associated stimuli in driving behavior, as observed in two different tasks: the CPP test, which measures food reward, and the second-order schedule of reinforcement, which measures food-seeking behavior. Evolutionarily, food, and in particular energy-dense highly palatable food, has the ability to increase the salience of associated contextual stimuli. Through this mechanism, contextual stimuli can, therefore, exert a strong control over behavior even in absence of food per se, and in pathological conditions they have the power to initiate and sustain binge eating (Giuliano and Cottone, 2015). Our results show that tactile and visual cues associated with the highly palatable food were able to induce a strong place preference, unlike stimuli associated to the standard chow diet. We observed that RO5256390 treatment fully and selectively blocked the expression of $\mathrm{CPP}$ in rats of the Palatable food group. Furthermore, a palatable food-associated stimulus light was able to induce a vigorous food-seeking behavior in a second-order schedule of reinforcement (Everitt and Robbins, 2000), which was fully and selectively blocked by RO5256390 treatment. Importantly, the drug effect on food seeking was observed during the first interval, and, therefore, before food ingestion occurred, suggesting an effect on incentive value of palatable food. Once again, we can confidently exclude that this effect was induced by an overall behavioral deficit induced by the drug as inactive lever responding was unaffected. In addition, responding for the cue associated with the standard chow diet was not affected by drug treatment.

\section{Role of mPFC TAAR1 in Binge-like Eating of Palatable Food}

In this study, we found that binge-like eating of palatable food decreased TAAR1 protein levels in the MPFC. The $\mathrm{mPFC}$ is a key area involved in inhibitory control and decision making, and dysfunctions of this region are thought to contribute to compulsive, binge-like eating behavior (Blasio et al, 2014; Calu et al, 2013; Cottone et al, 2012; Mena et al, 2013). TAAR1 KO mice have been shown to display behaviors consistent with cortical dysfunctions, such 
as perseverative behavior and impulsivity; consistently, TAAR1 agonism improves these behaviors in wild-type animals (Espinoza et al, 2015), suggesting that a deficiency in TAAR1, like the one we observed in the mPFC of rats in the Palatable food group, may result in deficits in cognitive function and behavioral flexibility. Therefore, it can be hypothesized that TAAR1 agonism may reduce compulsive, binge eating of palatable food by normalizing the palatableinduced impairment in TAAR1 signaling in the mPFC. In support of this hypothesis, as well as of a functional role of the TAAR1 protein reduction observed in $\mathrm{MPFC}$, is our finding that intra-mPFC administration of the TAAR1 agonist decreased binge-like eating in rats from the Palatable food group, without affecting the intake of regular chow. Interestingly, only intra-IL (and not intra-PrL) administration of TAAR1 reduced intake in binge eating rats, supporting the widely accepted notion that these two subregions have separable roles in behavior due to their very distinct connectivity. It should be acknowledged that, although unlikely, a potential explanation for the differential effect of RO5256390 microinfusion within either the IL or the PrL cortices may be the large variability of operant responding under vehicle conditions in the two cohorts of rats. Of relevance in this context are the observations that the IL has been shown to have elevated basal dopamine levels as compared with $\operatorname{PrL}$, potentially consistent with higher dopaminergic innervation, and that the IL subregion is crucial for the development and expression of inflexible reward seeking, as well as habitual behavior (Barker et al, 2014). To be noted is that the degree of reduction observed following intra-IL RO5256390 microinfusion was lower than that observed following intraperitoneal administration of the drug, suggesting that other TAAR1-expressing brain areas in addition to the IL may be contributing to the systemic drug effects (eg ventral tegmental area, dorsal raphe, and so on).

\section{CONCLUSIONS}

The TAAR1 system has been reported to regulate the behavioral actions of drugs of abuse. Indeed, consistent with our results, TAAR1 agonists were shown to be able to block cocaine seeking (Pei et al, 2014), suppress the reinforcing and rewarding effects of cocaine (Pei et al, 2015), attenuate cocaine behavioral sensitization, and reinstatement of cocaine seeking, as well as the expression of cocaineinduced CPP (Liu et al, 2016; Thorn et al, 2014). In line with the results from the pharmacological studies, TAAR1 KO mice display heightened locomotor response and context-dependent locomotor sensitization to amphetamine, and increased sensitivity to reinstatement of amphetamineinduced CPP, and they consume more alcohol and methamphetamine, compared with wild-type mice (Harkness et al, 2015; Lindemann et al, 2008; Lynch et al, 2013; Sukhanov et al, 2016; Wolinsky et al, 2007). It is hypothesized that TAAR1 involvement on drug-related behaviors may occur through the modulation of dopaminergic transmission. Indeed, TAAR1 knockout mice display a spontaneously elevated firing rate of dopaminergic neurons in the ventral tegmental area (Lindemann et al, 2008), and TAAR1 agonists have been shown to suppress the firing of dopamine neurons (Bradaia et al, 2009; Lindemann et al,
2008; Revel et al, 2011), suggesting that endogenous TAAR1 activation may dampen dopaminergic neuronal firing.

Therefore, here it can be speculated that the effects of TAAR1 agonism on food reward may occur through modulation of the dopaminergic terminals in the mPFC. Palatable food consumption activates the reward circuitry and releases dopamine in the mPFC (Babbs et al, 2013; Volkow et al, 2008). In addition, limited access to palatable food results in neuroadaptations in the reward circuitries, which contribute to compulsive overeating (Volkow et al, 2013). Moreover, obese and binge eating individuals display sensitized responses to conditioned food cues, such as increased prefrontal activation as well as higher dopamine release when exposed to food cues (Dimitropoulos et al, 2012). Hence, the observed effects of TAAR1 activation on food reward may be due to the ability of RO5256390 treatment to restore an impaired prefrontocortical dopaminergic transmission induced by excessive consumption of highly palatable food, although additional studies will be needed to confirm this hypothesis.

In summary, our results substantiate the potential for RO5256390 as a pharmacological treatment for disorders characterized by compulsive, binge eating.

\section{FUNDING AND DISCLOSURE}

This publication was made possible by grant numbers DA030425, MH091945, and MH093650 from the National Institute on Drug Abuse (NIDA), and the National Institute of Mental Health (NIMH), by the Peter Paul Career Development Professorship (PC), the McManus Charitable Trust (VS), and Boston University's Undergraduate Research Opportunities Program (UROP). Its contents are solely the responsibility of the authors and do not necessarily represent the official views of the National Institutes of Health. MCH is an employee of F. Hoffmann-La Roche. The remaining authors declare no potential conflict of interest.

\section{ACKNOWLEDGMENTS}

We thank Clara Velázquez-Sánchez, Lillian Huang, Shilpa Prasad, Jesse Mendoza, and Rachel Leavitt for technical and editorial assistance. We also thank Dr Roger Norcross for the synthesis of the TAAR1 agonist RO5256390.

\section{REFERENCES}

APA (2013). Diagnostic and Statistical Manual of Mental Disorders 5th edn. American Psychiatric Association.

Babbs RK, Unger EL, Corwin RL (2013). 2-Hydroxyestradiol enhances binge onset in female rats and reduces prefrontal cortical dopamine in male rats. Horm Behav 63: 88-96.

Barker JM, Taylor JR, Chandler LJ (2014). A unifying model of the role of the infralimbic cortex in extinction and habits. Learn Mem 21: $441-448$.

Blasio A, Steardo L, Sabino V, Cottone P (2014). Opioid system in the medial prefrontal cortex mediates binge-like eating. Addict Biol 19: 652-662.

Borowsky B, Adham N, Jones KA, Raddatz R, Artymyshyn R, Ogozalek KL et al (2001). Trace amines: identification of a family of mammalian G protein-coupled receptors. Proc Natl Acad Sci USA 98: 8966-8971. 
Bradaia A, Trube G, Stalder H, Norcross RD, Ozmen L, Wettstein JG et al (2009). The selective antagonist EPPTB reveals TAAR1mediated regulatory mechanisms in dopaminergic neurons of the mesolimbic system. Proc Natl Acad Sci USA 106: 20081-20086.

Bunzow JR, Sonders MS, Arttamangkul S, Harrison LM, Zhang G, Quigley DI et al (2001). Amphetamine, 3,4-methylenedioxymethamphetamine, lysergic acid diethylamide, and metabolites of the catecholamine neurotransmitters are agonists of a rat trace amine receptor. Mol Pharmacol 60: 1181-1188.

Burchett SA, Hicks TP (2006). The mysterious trace amines: protean neuromodulators of synaptic transmission in mammalian brain. Prog Neurobiol 79: 223-246.

Calu DJ, Kawa AB, Marchant NJ, Navarre BM, Henderson MJ, Chen B et al (2013). Optogenetic inhibition of dorsal medial prefrontal cortex attenuates stress-induced reinstatement of palatable food seeking in female rats. J Neurosci 33: 214-226.

Cottone P, Sabino V, Steardo L, Zorrilla EP (2007). FG 7142 specifically reduces meal size and the rate and regularity of sustained feeding in female rats: evidence that benzodiazepine inverse agonists reduce food palatability. Neuropsychopharmacology 32: 1069-1081.

Cottone P, Sabino V, Steardo L, Zorrilla EP (2008). Intermittent access to preferred food reduces the reinforcing efficacy of chow in rats. Am J Physiol Regul Integr Comp Physiol 295: R1066-R1076.

Cottone P, Sabino V, Steardo L, Zorrilla EP (2009). Consummatory, anxiety-related and metabolic adaptations in female rats with alternating access to preferred food. Psychoneuroendocrinology 34: $38-49$.

Cottone P, Wang X, Park JW, Valenza M, Blasio A, Kwak J et al (2012). Antagonism of sigma-1 receptors blocks compulsivelike eating. Neuropsychopharmacology 37: 2593-2604.

D'Cunha TM, Sedki F, Macri J, Casola C, Shalev U (2013). The effects of chronic food restriction on cue-induced heroin seeking in abstinent male rats. Psychopharmacology (Berl) 225: 241-250.

Dimitropoulos A, Tkach J, Ho A, Kennedy J (2012). Greater corticolimbic activation to high-calorie food cues after eating in obese vs. normal-weight adults. Appetite 58: 303-312.

Dore R, Iemolo A, Smith KL, Wang X, Cottone P, Sabino V (2013). CRF mediates the anxiogenic and anti-rewarding, but not the anorectic effects of PACAP. Neuropsychopharmacology 38: $2160-2169$.

Dore R, Valenza M, Wang X, Rice KC, Sabino V, Cottone P (2014). The inverse agonist of CB1 receptor SR141716 blocks compulsive eating of palatable food. Addict Biol 19: 849-861.

Espinoza S, Lignani G, Caffino L, Maggi S, Sukhanov I, Leo D et al (2015). TAAR1 Modulates Cortical Glutamate NMDA Receptor Function. Neuropsychopharmacology 40: 2217-2227.

Everitt BJ, Robbins TW (2000). Second-order schedules of drug reinforcement in rats and monkeys: measurement of reinforcing efficacy and drug-seeking behaviour. Psychopharmacology (Berl) 153: $17-30$.

Gearhardt AN, Boswell RG, White MA (2014). The association of 'food addiction' with disordered eating and body mass index. Eat Behav 15: 427-433.

Giuliano C, Cottone P (2015). The role of the opioid system in binge eating disorder. CNS Spectr 20: 537-545.

Grandy DK, Miller GM, Li JX (2016). 'TAARgeting Addiction'-The Alamo Bears Witness to Another Revolution: An Overview of the Plenary Symposium of the 2015 Behavior, Biology and Chemistry Conference. Drug Alcohol Depend 159: 9-16.

Harkness JH, Shi X, Janowsky A, Phillips TJ (2015). Trace amineassociated receptor 1 regulation of methamphetamine intake and related traits. Neuropsychopharmacology 40: 2175-2184.

Harmeier A, Obermueller S, Meyer CA, Revel FG, Buchy D, Chaboz $S$ et al (2015). Trace amine-associated receptor 1 activation silences GSK3beta signaling of TAAR1 and D2R heteromers. Eur Neuropsychopharmacol 25: 2049-2061.
Iemolo A, Ferragud A, Cottone P, Sabino V (2015). Pituitary adenylate cyclase-activating peptide in the central amygdala causes anorexia and body weight loss via the melanocortin and the TrkB systems. Neuropsychopharmacology 40: $1846-1855$.

Lindemann L, Hoener MC (2005). A renaissance in trace amines inspired by a novel GPCR family. Trends Pharmacol Sci 26: 274-281.

Lindemann L, Meyer CA, Jeanneau K, Bradaia A, Ozmen L, Bluethmann $\mathrm{H}$ et al (2008). Trace amine-associated receptor 1 modulates dopaminergic activity. J Pharmacol Exp Ther 324: 948-956.

Liu JF, Thorn DA, Zhang Y, Li JX (2016). Effects of trace amineassociated receptor 1 agonists on the expression, reconsolidation, and extinction of cocaine reward memory. Int J Neuropsychopharmacol 19: pyw009.

Lynch LJ, Sullivan KA, Vallender EJ, Rowlett JK, Platt DM, Miller GM (2013). Trace amine associated receptor 1 modulates behavioral effects of ethanol. Subst Abuse 7: 117-126.

Mena JD, Selleck RA, Baldo BA (2013). Mu-opioid stimulation in rat prefrontal cortex engages hypothalamic orexin/hypocretincontaining neurons, and reveals dissociable roles of nucleus accumbens and hypothalamus in cortically driven feeding. J Neurosci 33: 18540-18552.

Michaelides M, Thanos PK, Volkow ND, Wang GJ (2012). Dopamine-related frontostriatal abnormalities in obesity and binge-eating disorder: emerging evidence for developmental psychopathology. Int Rev Psychiatry 24: 211-218.

Micioni Di Bonaventura MV, Ciccocioppo R, Romano A, Bossert JM, Rice KC, Ubaldi M et al (2014). Role of bed nucleus of the stria terminalis corticotrophin-releasing factor receptors in frustration stress-induced binge-like palatable food consumption in female rats with a history of food restriction. J Neurosci 34: 11316-11324.

Miller GM, Verrico CD, Jassen A, Konar M, Yang H, Panas H et al (2005). Primate trace amine receptor 1 modulation by the dopamine transporter. J Pharmacol Exp Ther 313: 983-994.

Murray S, Tulloch A, Gold MS, Avena NM (2014). Hormonal and neural mechanisms of food reward, eating behaviour and obesity. Nat Rev Endocrinol 10: 540-552.

Parylak SL, Cottone P, Sabino V, Rice KC, Zorrilla EP (2012). Effects of $\mathrm{CB} 1$ and $\mathrm{CRF} 1$ receptor antagonists on binge-like eating in rats with limited access to a sweet fat diet: lack of withdrawallike responses. Physiol Behav 107: 231-242.

Pei Y, Asif-Malik A, Canales JJ (2016). Trace amines and the trace amine-associated receptor 1: pharmacology, neurochemistry, and clinical implications. Front Neurosci 10: 148.

Pei Y, Lee J, Leo D, Gainetdinov RR, Hoener MC, Canales JJ (2014). Activation of the trace amine-associated receptor 1 prevents relapse to cocaine seeking. Neuropsychopharmacology 39: 2299-2308.

Pei Y, Mortas P, Hoener MC, Canales JJ (2015). Selective activation of the trace amine-associated receptor 1 decreases cocaine's reinforcing efficacy and prevents cocaine-induced changes in brain reward thresholds. Prog Neuropsychopharmacol Biol Psychiatry 63: 70-75.

Raab S, Wang H, Uhles S, Cole N, Alvarez-Sanchez R, Kunnecke B et al (2016). Incretin-like effects of small molecule trace amineassociated receptor 1 agonists. Mol Metab 5: 47-56.

Revel FG, Moreau JL, Gainetdinov RR, Bradaia A, Sotnikova TD, Mory R et al (2011). TAAR1 activation modulates monoaminergic neurotransmission, preventing hyperdopaminergic and hypoglutamatergic activity. Proc Natl Acad Sci USA 108: 8485-8490.

Revel FG, Moreau JL, Gainetdinov RR, Ferragud A, VelazquezSanchez C, Sotnikova TD et al (2012). Trace amine-associated receptor 1 partial agonism reveals novel paradigm for neuropsychiatric therapeutics. Biol Psychiatry 72: 934-942. 
Sabino V, Cottone P, Steardo L, Schmidhammer H, Zorrilla EP (2007). 14-Methoxymetopon, a highly potent mu opioid agonist, biphasically affects ethanol intake in Sardinian alcohol-preferring rats. Psychopharmacology 192: 537-546.

Seiglie MP, Smith KL, Blasio A, Cottone P, Sabino V (2015). Pituitary adenylate cyclase-activating polypeptide induces a depressive-like phenotype in rats. Psychopharmacology (Berl) 232: 3821-3831.

Smith KL, Rao RR, Velazquez-Sanchez C, Valenza M, Giuliano C, Everitt BJ et al (2015). The uncompetitive N-methyl-D-aspartate antagonist memantine reduces binge-like eating, food-seeking behavior, and compulsive eating: role of the nucleus accumbens shell. Neuropsychopharmacology 40: 1163-1171.

Sukhanov I, Caffino L, Efimova EV, Espinoza S, Sotnikova TD, Cervo L et al (2016). Increased context-dependent conditioning to amphetamine in mice lacking TAAR1. Pharmacol Res 103: 206-214.

Thorn DA, Jing L, Qiu Y, Gancarz-Kausch AM, Galuska CM, Dietz DM et al (2014). Effects of the trace amine-associated receptor 1 agonist RO5263397 on abuse-related effects of cocaine in rats. Neuropsychopharmacology 39: 2309-2316.

Velazquez-Sanchez C, Ferragud A, Moore CF, Everitt BJ, Sabino V, Cottone P (2014). High trait impulsivity predicts food addiction-like behavior in the rat. Neuropsychopharmacology 39: 2463-2472.

Velazquez-Sanchez C, Santos JW, Smith KL, Ferragud A, Sabino V, Cottone P (2015). Seeking behavior, place conditioning, and resistance to conditioned suppression of feeding in rats intermittently exposed to palatable food. Behav Neurosci 129: 219-224.

Volkow ND, Wang GJ, Fowler JS, Telang F (2008). Overlapping neuronal circuits in addiction and obesity: evidence of systems pathology. Philos Trans $R$ Soc Lond B Biol Sci 363: 3191-3200.

Volkow ND, Wang GJ, Tomasi D, Baler RD (2013). The addictive dimensionality of obesity. Biol Psychiatry 73: 811-818.

Wolinsky TD, Swanson CJ, Smith KE, Zhong H, Borowsky B, Seeman $\mathrm{P}$ et al (2007). The Trace Amine 1 receptor knockout mouse: an animal model with relevance to schizophrenia. Genes Brain Behav 6: 628-639.

Xie Z, Westmoreland SV, Bahn ME, Chen GL, Yang H, Vallender EJ et al (2007). Rhesus monkey trace amineassociated receptor 1 signaling: enhancement by monoamine transporters and attenuation by the $\mathrm{D} 2$ autoreceptor in vitro. J Pharmacol Exp Ther 321: 116-127.

Supplementary Information accompanies the paper on the Neuropsychopharmacology website (http://www.nature.com/npp) 